\title{
A STOCHASTIC MOBILE DATA TRAFFIC MODEL FOR VEHICULAR AD HOC NETWORKS
}

\section{Dr. S. Smys}

Professor, CSE, RVS Technical Campus,

Coimbatore, India

Email id: smys375@gmail.com

\section{Dr. Jennifer S. Raj}

Professor, ECE, Gnanamani college of Technology

Namakkal, India.

Email id: jennifer.raj@gmail.com

\begin{abstract}
Routing and mobile data traffic management is a major performance affecting issue in vehicular Ad Hoc networks (VANET). High-rise structures and such radio obstacles cause trouble in proper reception of signals when position-based routing schemes are used. Other major challenges include constrained mobility and irregular distribution of vehicular nodes. A stochastic mobile data traffic model is presented in this paper. This model offers security, reliability, safety and comfort for driving by overcoming the problems of traffic congestion, interference and jamming. It also addresses the handover (HO) issue that occurs during fast mobility. Along with this, the quality parameters of the system such as throughput, packet delivery ratio and delay are also evaluated.
\end{abstract}

Keywords: Ad Hoc Networks, VANET, Handover, EAODV

\section{INTRODUCTION}

A Mobile Adhoc Network (MANET) scheme in which automobiles form the mobile nodes is termed as Vehicular Adhoc Network (VANET) [5]. Instead of moving in random, the highly mobile nodes of VANET has a unique feature of following pre-structured routes. Vehicles communicate with Base Station, Road-Side-Units (RSUs), as well as with each other. The VANET based automobiles perform Wireless Access in Vehicular Environment (WAVE) using ratio transceivers, Global Positioning System (GPS) based localization, sensor and processing unit equipped On-Board Units (OBUs), and make use of various such cutting-edge technologies to form an Intelligent Transportation System (ITS). 
Vol.01/ No. 01

Pages: 55-63

https://www.irojournals.com/jucct/

DOI: https://doi.org/10.36548/jucct.2019.1.006

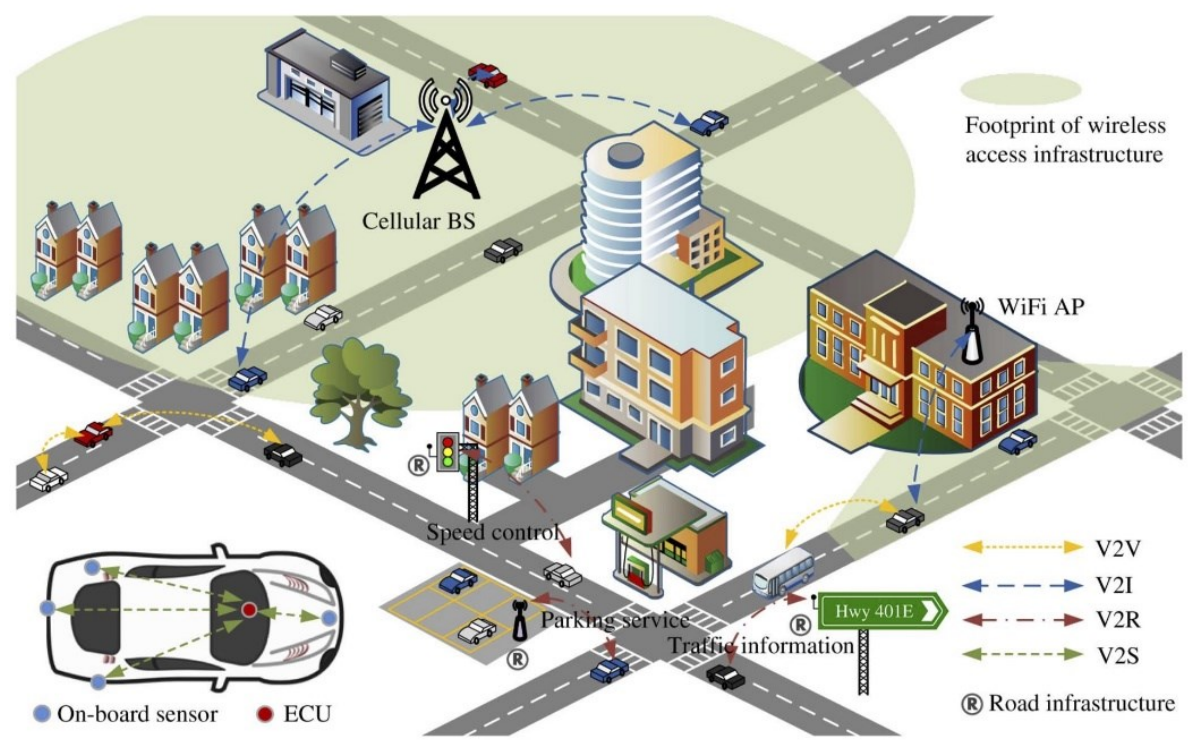

Figure 1: VANET Architecture [7]

The major characteristics of VANET include - low power consumption, lane-constrained mobility patterns, fast time-varying channel, varying vehicle count with correlated or autonomous speeds, time-varying topology and location prediction oriented trajectory-based movements [4]. Due to the exceptional trace support, this work makes use of VanetMobiSim software for traffic model and vehicular mobility generation. NS-2 simulator is used for network simulation and Trans for VANET Simulation. The foremost problems in mobile VANET systems include mobility handover issue, communication interference and jamming. Figure 2 represents the various categories of handover in VANETs.

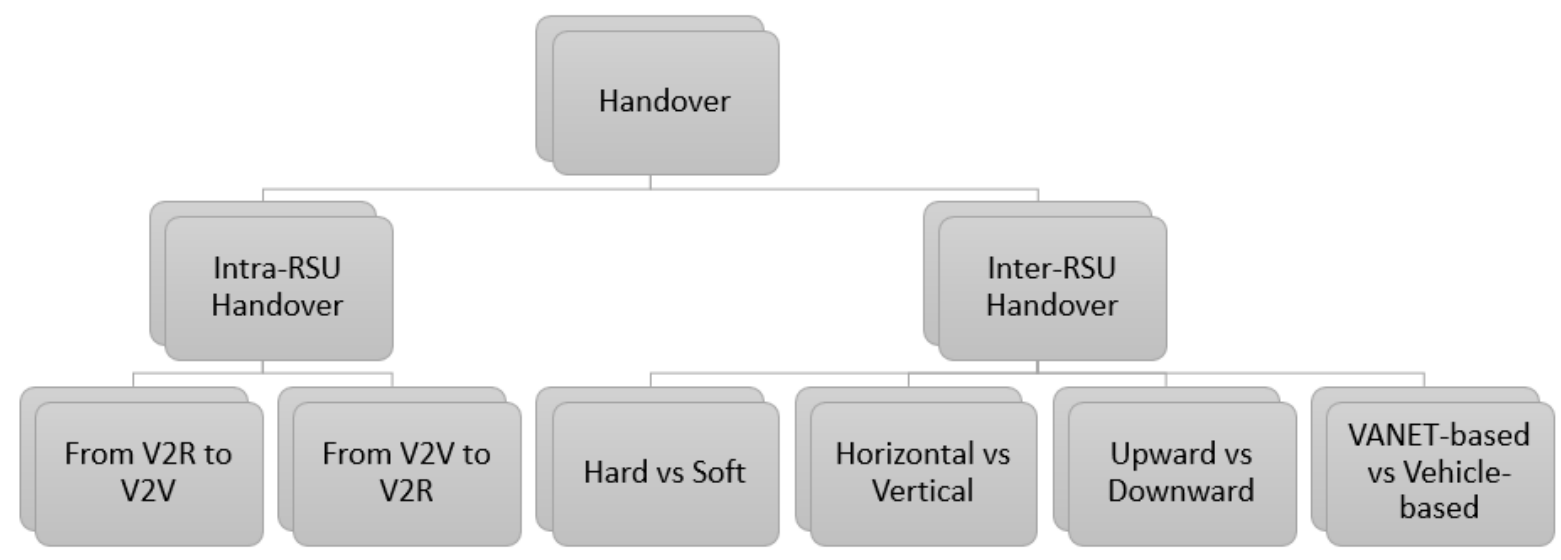

Figure 2: Handover Classification 
When the connection of a vehicle is changed to RSU (V2R) from vehicle (V2V), handover occurs. This and the vice versa are termed as Intra-RSU handovers. In case of inter-RSU handovers, 4 different types of handovers exist namely VANET based vs vehicle based, Upward vs Downward, Horizontal vs Vertical, and Hard vs Soft.

\section{EXISTING LITERATURE}

Chunfeng Liu et al [1] performed neighbour discovery with Kalman prediction model in VANET. This method used spatial and temporal features of the nodes to offer an efficient, robust and accurate simulation. The algorithm outperforms ROMSG, Autoregressive Hello (ARH), hello protocol in AODV (HP-AODV) and such traditional protocols. It reduces the average error rate by $15 \%$. Adel Ahmed et al [2] extensively analysed the VANET based handover management in $5 \mathrm{G}$ network. The paper proposed solutions in terms of predictive mathematical model, channel characteristic model, vehicle velocity and Mobile IP integration into VANET.

The major issues causing failure of $\mathrm{HO}$ are classified as data traffic congestion, coverage hole, communication interference and jamming and mobility. Unnecessary HO, Ping-Pong HO, HO to wrong cell, tooearly $\mathrm{HO}$ and too-late $\mathrm{HO}$ are the categories of mobility $\mathrm{HO}$ issues. Software-defined networking (SDN) architecture is a plausible solution to these issues. Parveen et al [3] proposed an Enhanced AODV (ENAODV) model such that it performs better than the traditional AODV method and offers better efficiency. The system QoS performance and network parameters are analysed.

Francisco et al [4] surveyed and compared various VANET simulators inclusive of Simulation of Urban Mobility (SUMO), VanetMobiSim, MObility model generator for VEhicular networks (MOVE), STreet RAndom Waypoint (STRAW), FreeSim, CityMob, and so on. Further, network simulators like NS-2, GloMoSim, JiST/SWANS and SNS are analysed along with VANET simulators like Traffic and Network Simulation Environment (TraNS), GrooveNet, National Chiao Tung University Network Simulator (NCTU) and MobiREAL. 


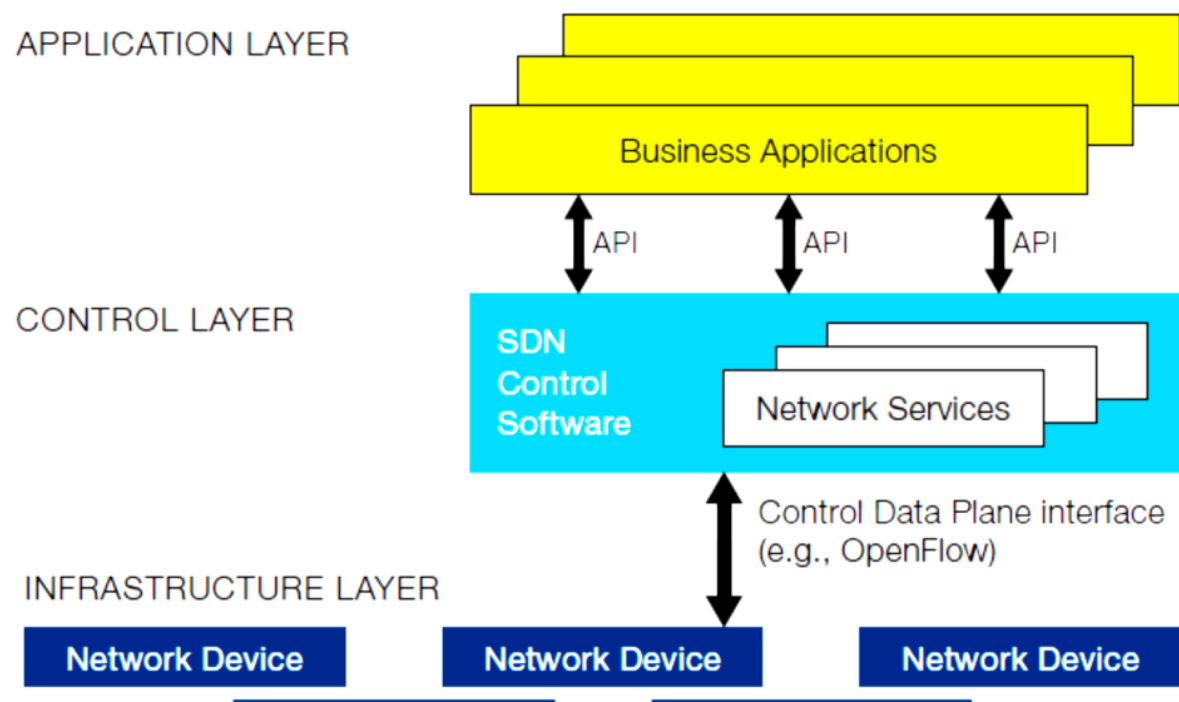

Figure 3: SDN Architecture [5]

Nguyen B.Truong et al [5] proposed an SDN based VANET with fog computing. This combination offers location-awareness service, delay-sensitivity, global knowledge, programmability, scalability and flexibility. Mobility support, location awareness and low latency are the major features of fog computing. Darwish et al [6] performed a review for estimating traffic density in VANET. The paper compared estimation based on grouping or clustering, V2R and VANET communication as well as traffic flow information. The issues in such as maintenance and deployment cost as well as coverage issues are discussed.

Tamer et al [8] analysed the VANET based data distribution models such as - same-directional, opposite directional and bi-directional dissemination model. Broadcast utilization rate, accuracy and latency time are the chief factors analysed for this purpose. The comparison is quantitatively expressed through latency, accuracy and knowledge graphs. Huixian Wang et al [9] implemented VANET based cluster design, comparison and model validation, contention window design and traffic control using a new analytical model. Interference management, channel allocation and inter-cluster routing are future avenues for this research.

Salim Bitam et al [10] introduced "VANET-Cloud: A Generic Cloud Computing Model for Vehicular Ad Hoc Networks". The cloud challenges such as privacy and security issues, sensing and aggregation data, Green VANET cloud, coordination and communication between the cloud sub-models are addressed. Banoth Ravi et al [11] "calculated the average individual delay using queuing theory technique of stochastic analysis approach and the deterministic analysis approach for vehicular ad-hoc networks." 
Shangguang Wang et al [12] performed "Offloading mobile data traffic for QoS-aware service provision in vehicular cyber-physical systems". The efficiency of this system is optimal even for homogeneous automobiles. Moumena Chaqfeh et al [3] performed a VANET based data dissemination. The push, pull and hybrid models are discussed in detail. Kalaivani Dhanasegaran et al [15] introduced "DCDS: Data centric dispatcher selection protocol for cellular enabled VANET communication". Tao Lei et al [16] developed "AOM: adaptive mobile data traffic offloading for M2M networks".

\section{PROPOSED WORK}

We use an Enhanced Ad Hoc on Demand Distance Vector (EADOV) Routing Protocol. When a node is ready to transmit data packets, it simultaneously starts the process of route maintenance and discovery. When data packets fail to be delivered to the destination node, it is broadcasted to its neighbour. The destination note establishes a reverse path for the sender node such that the previous nodes lowest lifetime is maximised. Route table management, route discovery, forward and reverse-path setup and route maintenance are the major features are analysed in this regard. Forward and backward routing tables are established containing soft states. The sample routing tables are as shown below.

Table I - Forward Routing Table

\begin{tabular}{|c|c|c|c|c|c|c|}
\hline \multicolumn{6}{|c|}{ FIELDS OF ENTRY } \\
\hline Destination & Next Hop & Hop Count & Destination & Active & Expire & Transmit \\
Address & & & Sequence & Neighbour & time & Power \\
& & & Number & & & \\
\hline
\end{tabular}

Table II - Backward Routing Table

\begin{tabular}{|c|c|c|c|c|c|c|c|}
\hline \multicolumn{2}{|c|}{ FIELDS OF ENTRY } \\
\hline Destination & Next Hop & Hop Count & Destination & Active & Expire & Route & Received \\
Address & & & Sequence & Neighbour & time & Lifetime & power \\
& & & Number & & & & \\
\hline
\end{tabular}

Route discovery is done while sending data packets to a node without destination path. Next, the forward and reverse path are setup. In Table I and II, are based on sending data. It contains information of the destination address, expire time, active neighbours, destination sequence number, hop count, and so on. These routing tables are managed with the help of a timer. We can calculate the routing cost using the following expression 
Journal of Ubiquitous Computing and Communication Technologies (UCCT) (2019)

Vol.01/ No. 01

Pages: 55-63

https://www.irojournals.com/jucct/

DOI: https://doi.org/10.36548/jucct.2019.1.006

$$
R C=\frac{\text { Hello }_{n}+O_{n}}{P_{n}}
$$

Where $\mathrm{O}_{n}=$ routing control messages, Hello $n=$ Hello message count, $\mathrm{P}_{\mathrm{n}}=$ Total throughput. $\mathrm{O}_{\mathrm{n}}$ consists of routing error (RERR), Routing Response (RREP) and Routing Request Message (RREQ).

\section{RESULT}

In order to create a realistic VANET simulation by integrating network simulator and mobility generator, we use Traffic and Network Simulation Environment (TraNS) tool. It is an open source software and works based on $\mathrm{C}++$ and Java. Factors such as packet delivery ratio (PDR), End-to-End Delay (EED) and throughput are measured and compared with the AODV and proposed method.

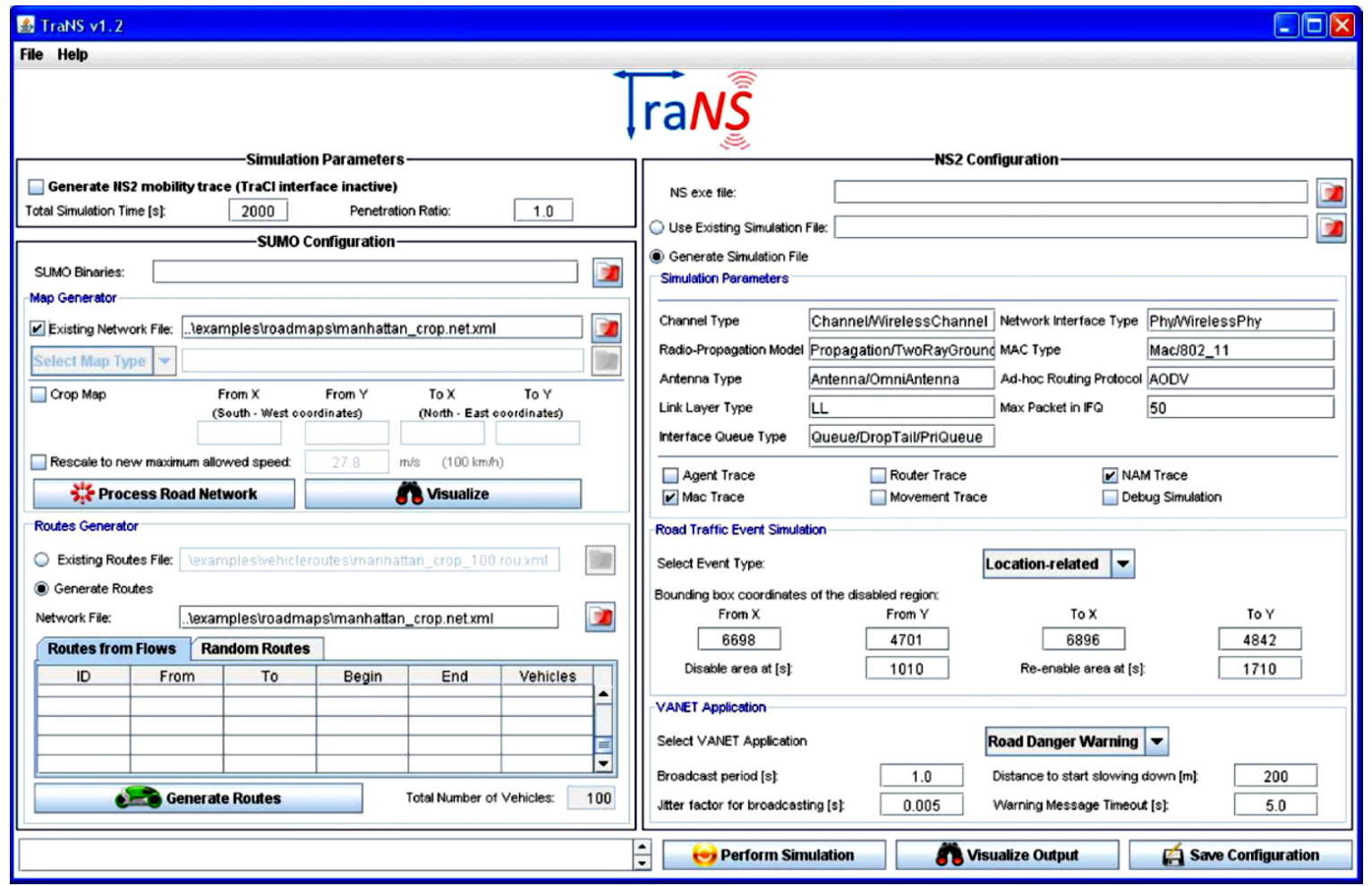

Figure 3: TraNS Graphical Interface 
Journal of Ubiquitous Computing and Communication Technologies (UCCT) (2019)

Vol.01/ No. 01

Pages: 55-63

https://www.irojournals.com/jucct/

DOI: https://doi.org/10.36548/jucct.2019.1.006

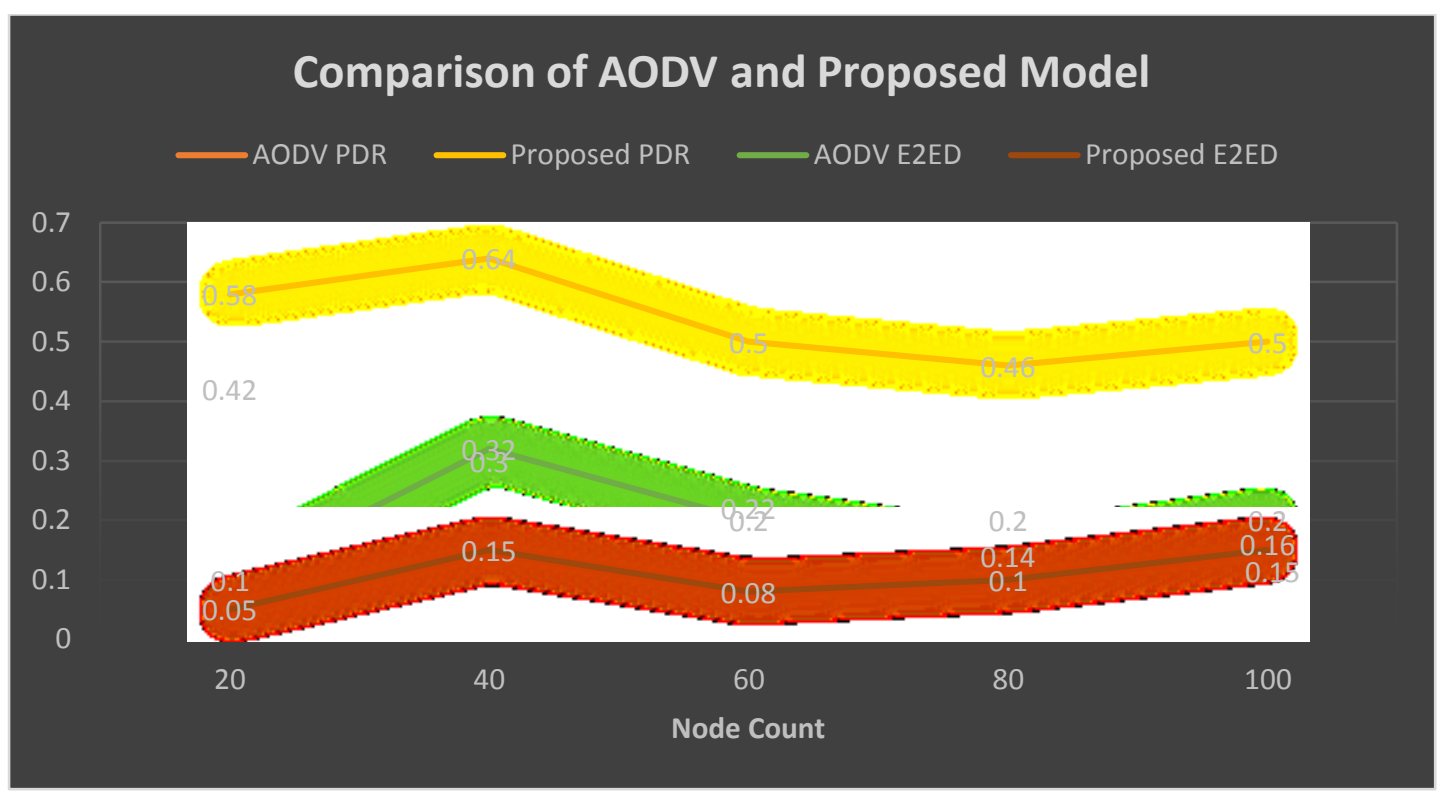

Figure 4: Comparison of AODV and Proposed Model

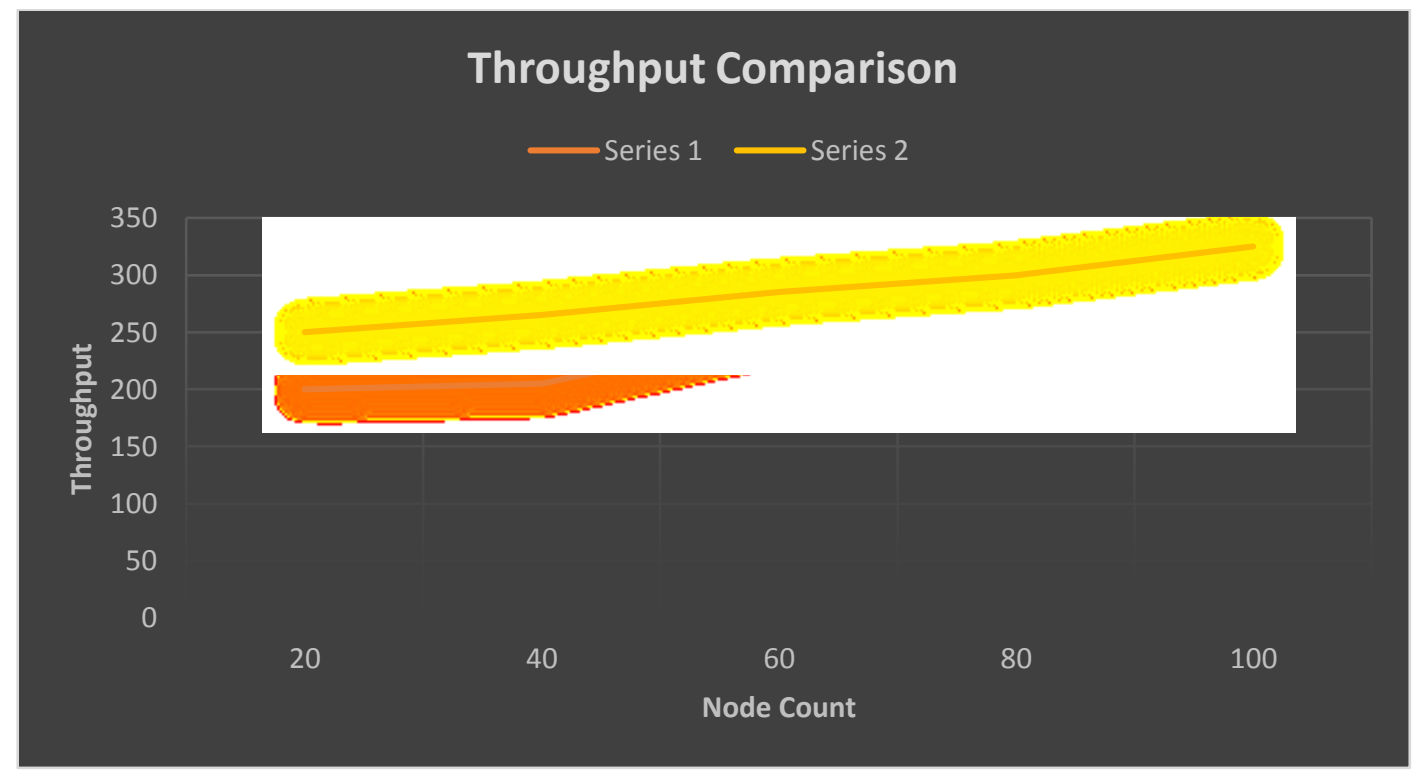

Figure 5: Throughput Comparison

From figure 4 and 5 , the performance of the system varies based on the number of nodes. The proposed method is efficient when compared to the traditional AODV technique.

\section{CONCLUSION AND FUTURE SCOPE}


Journal of Ubiquitous Computing and Communication Technologies (UCCT) (2019)

Vol.01/ No. 01

Pages: 55-63

https://www.irojournals.com/jucct/

DOI: https://doi.org/10.36548/jucct.2019.1.006

The proposed method improves the performance and offers an enhanced mobile data traffic model for VANET. We use NS-2 and TraNS for simulation purpose and analyse the performance based on throughput, PDR and EED. The proposed system reduces the overhead and packet loss and improves the packet delivery and delay. Future work will be based on real-world implementation, testing and improvement of the proposed method. It also offers better reliability, safety and comfort and addresses the handover issue found in traditional methods.

\section{References}

[1] Liu, Chunfeng, Gang Zhang, Weisi Guo, and Ran He. "Kalman Prediction-Based Neighbor Discovery and Its Effect on Routing Protocol in Vehicular Ad Hoc Networks." IEEE Transactions on Intelligent Transportation Systems (2019).

[2] Ahmed, Adel A., and Ahmad A. Alzahrani. "A comprehensive survey on handover management for vehicular ad hoc network based on 5G mobile networks technology." Transactions on Emerging Telecommunications Technologies 30, no. 3 (2019): e3546.

[3] Singhal, Parveen Kumar, and V. K. Chaubey. "An Enhanced Ad Hoc on Demand Distance Vector Routing Protocol for Vehicular Ad Hoc Networks (VANET's)." Available at SSRN 3355137 (2019).

[4] Martinez, Francisco J., Chai Keong Toh, Juan- Carlos Cano, Carlos T. Calafate, and Pietro Manzoni. "A survey and comparative study of simulators for vehicular ad hoc networks (VANETs)." Wireless Communications and Mobile Computing 11, no. 7 (2011): 813-828.

[5] Truong, Nguyen B., Gyu Myoung Lee, and Yacine Ghamri-Doudane. "Software defined networkingbased vehicular adhoc network with fog computing." In 2015 IFIP/IEEE International Symposium on Integrated Network Management (IM), pp. 1202-1207. IEEE, 2015.

[6] Darwish, Tasneem, and K. Abu Bakar. "Traffic density estimation in vehicular ad hoc networks: A review." Ad Hoc Networks 24 (2015): 337-351.

[7] https://ece.uwaterloo.ca/ kan.yang/security_bbcr/vanet.html

[8] Nadeem, Tamer, Pravin Shankar, and Liviu Iftode. "A comparative study of data dissemination models for VANETs." In 2006 Third Annual International Conference on Mobile and Ubiquitous Systems: Networking \& Services, pp. 1-10. IEEE, 2006.

[9] Wang, Huixian, Ren Ping Liu, Wei Ni, Wei Chen, and Iain B. Collings. "VANET modeling and clustering design under practical traffic, channel and mobility conditions." IEEE Transactions on Communications 63, no. 3 (2015): 870-881.

[10]Bitam, Salim, Abdelhamid Mellouk, and Sherali Zeadally. "VANET-cloud: a generic cloud computing model for vehicular Ad Hoc networks." IEEE Wireless Communications 22, no. 1 (2015): 96-102.

[11] Ravi, Banoth, Jaisingh Thangaraj, and Shrinivas Petale. "Data Traffic Forwarding for Inter-vehicular Communication in VANETs Using Stochastic Method." Wireless Personal Communications 106, no. 3 (2019): 1591-1607. 
Journal of Ubiquitous Computing and Communication Technologies (UCCT) (2019)

Vol.01/ No. 01

Pages: 55-63

https://www.irojournals.com/jucct/

DOI: https://doi.org/10.36548/jucct.2019.1.006

[12] Wang, Shangguang, Tao Lei, Lingyan Zhang, Ching-Hsien Hsu, and Fangchun Yang. "Offloading mobile data traffic for QoS-aware service provision in vehicular cyber-physical systems." Future Generation Computer Systems 61 (2016): 118-127.

[13] Chaqfeh, Moumena, Abderrahmane Lakas, and Imad Jawhar. "A survey on data dissemination in vehicular ad hoc networks." Vehicular Communications 1, no. 4 (2014): 214-225.

[14] el Mouna Zhioua, Ghayet, Jun Zhang, Houda Labiod, Nabil Tabbane, and Sami Tabbane. "VOPP: A VANET offloading potential prediction model." In 2014 IEEE Wireless Communications and Networking Conference (WCNC), pp. 2408-2413. IEEE, 2014.

[15] Dhanasegaran, Kalaivani, and Rajkumar Soundrapandiyan. "DCDS: Data centric dispatcher selection protocol for cellular enabled VANET communication." International Journal of Communication Systems (2019): e3936.

[16]Lei, Tao, Shangguang Wang, Jinglin Li, and Fangchun Yang. "AOM: adaptive mobile data traffic offloading for M2M networks." Personal and Ubiquitous Computing 20, no. 6 (2016): 863-873. 